\title{
Effects of Pressure Soaking on Elastic Modulus of Steamed-Cooked Glutinous Rice Kernel
}

\author{
Parichat Glangchai and Wetchayan Rangsri* \\ Department of Mechanical Engineering, Faculty of Engineering, Chiang Mai University, \\ Chiang Mai 50200, Thailand
}

('Corresponding author's e-mail: w.rangsri@gmail.com)

Received: 10 August 2020, Revised: 16 June 2021, Accepted: 26 June 2021

\begin{abstract}
The goal of this research is to assess the moduli of elasticity of steamed-cooked Thai Sanpatong 1 glutinous rice (GR) under various soaking conditions, primarily normal soaking and pressure soaking. The soaking process can influence properties of cooked rice. One of the main objectives is to determine the stiffness of cooked rice after soaking with either of the 2 methods, ASAE 368.4 was employed as main protocol for property testing and then Hooke's and Hertz's equations were used to estimate the moduli of elasticity. Poisson's equation was used to estimate the Poisson's ratios. The results show that the moduli of elasticity of the cooked normal-soaked and pressure-soaked rice determined using Hooke's theory were 3.77 and $5.67 \mathrm{MPa}$, respectively. The moduli of elasticity of the cooked normal-soaked and pressure-soaked rice determined using Hertz's theory were 1.58 and $2.33 \mathrm{MPa}$, respectively. Poisson's ratio of the cooked normal-soaked and pressure-soaked rice were 0.27 and 0.28 , respectively. Results revealed that the pressure treated rice has higher stiffness.
\end{abstract}

Keywords: Young's Modulus, Bio-yield stress, Rigid plate compression, Water uptake, Thai rice

\section{Introduction}

Glutinous rice (sticky rice) is widely consumed. One of the most popular glutinous rice variety in Thailand's north and northeast regions, also recommended by the Thailand Ministry of Agriculture and Cooperatives, is the Sanpatong 1 (SPTLR84051-32-2-2-4) rice. This rice type has very low light sensitivity, high productivity, stable harvesting time, and can be grown throughout the year.

The traditional method of steaming requires soaking the rice in water overnight, which can take up to $12 \mathrm{~h}$, before streaming. Soaking time is inconvenient for restaurants, especially when demand is high. Attempts have been made to shorten the soaking period. Some researchers, such as Ahromrit [1], have demonstrated that this can be accomplished by soaking the rice under pressure, causing the rice to saturate more quickly and reduce soaking time. However, cooked rice obtained from reducing soaking time may respond to chewing unlike that obtained from traditional soaking. Sticky rice consumers in Thailand prefer to eat sticky rice that is soft. The softness of rice can be identified by mechanical properties such as modulus of elasticity. It is necessary to study the mechanical properties of rice that has undergone different soaking processes.

Iwasaki [2] divided the methods for measuring the texture quality of cooked rice into 2 categories: Sensory test and instrumentation test. From a sensory test - i.e., assessment by human senses by a panel of evaluators, wherein test results are recorded based on their responses to the products under test. It had been shown that the rice cooked by this method did not have any difference in stickiness to that was cooked by the traditional way. That said, the sensory test has its limitations, such as the uncertainty due to the taste of the person who takes the survey. These have to be taken into account when drawing a conclusion from a sensory test. Some researcher such as Mindo [3] studied the sensory assessment of cooked milled rice. This study proposes the use of quantitative method, namely compression test, to evaluate the difference of rice soaked by the described methods. Mechanical properties, i.e., modulus of elasticity and Poisson's ratio, during applied compression load under various conditions are compared and evaluated.

Perez [4] studied a factor affecting cooking time and the relationship between water-rice ratio in the rice cooker method and hardness of cooked rice using 10 milled rice samples. The puncture test was used 
s properties of rice. However, this method may not be ideal because it only determines mechanical behaviour in 1 area of each rice kernel. Using a different method, Shitanda [5] determined the Poisson's ratio and young's modulus of rough rice in their natural state under contact load between 2 rigid plates. Stress was determined by considering the variation of contact area determined by a piece of pressure sensitive paper placed on the compressing surface before the compression test. Burubai [6] determined the Poisson's ratios and moduli of elasticity of African nutmeg. Several methods have been suggested for measuring the elastic moduli of small pieces of agricultural materials using compression tests. The compressive modulus of elasticity was calculated by using the equation based on Hertz's solution to problems involving contact stresses recommended by the ASAE [7] Standard on contact between parallel plates. This Standard is intended for use in determining mechanical attributes resistance to mechanical injury as a result of static loading, and quasi-static force-deformation behavior [3] of food materials of convex shape, such as fruits and vegetables, seeds and grains, and manufactured food materials. Bamrungwong [8] studied mechanical properties of brown and milled rice kernels of 2 long grain Indica varieties and 2 japonica varieties. Under controlled atmospheric conditions of $20{ }^{\circ} \mathrm{C}$ and $33.0-75.5$ per cent RH, the kernels were subjected to lateral contact-point compression. They reported the breaking loads, rupture forces, and moduli of elasticity of long grain rice. The compressive moduli of elasticity were calculated using an equation based on Hertz's solution to contact stress problems, as recommended by the ASAE [7] Standard. Mostafa [9] studied the Poisson's ratio and Young's modulus of red bean grains and found that Young's modulus decreased as moisture content increased and increased with increasing loading rates. Osvaldo [10] determined the proportional deformity modulus of rough and dehulled rice grains for different moisture contents under a compression test. Manizhe [11] evaluated 3 theories (Hooke, Hertz and Boussinesq) on the apparent moduli of elasticity of some apple in order to estimate and predict the deformation of viscoelastic materials under uniaxial compression for system design, transport, processing and packaging.

The results of this study will inform the glutinous rice cooker about the effect on softness of glutinous rice when soaking glutinous rice under pressure. In addition, from the point of view of mechanics of materials, it will also reveal a suitable method for calculating the moduli of elasticity and Poisson's ratio of glutinous rice. These values can be used to validate the results of traditional sensory test. In subsequent studies, we propose to use the obtained properties to simulate the glutinous rice deformation behaviour under chewing situations. This method will enable rice quality to be evaluated in a variety of ways and conditions promptly, reducing the time and cost of the experiment process.

\section{Materials and methods}

\section{Glutinous rice samples}

Glutinous Rice, GR (Oryza sativa L. cv. Sanpatong 1) grown in Chiang Mai Province, Thailand, was tested. All of the kernels were obtained steamed-cooked. Gelatinized and retrograded glutinous rice is referred to as steamed-cooked glutinous rice. The starch in the rice becomes clear gel as a result of this process, and no visible white areas can be seen with the naked eye. The kernels were assumed to be isotropic and linear elastic. This is supported by the molecular structure of rice, which shows that starch distribution is consistent with no definite direction (Figure 1).
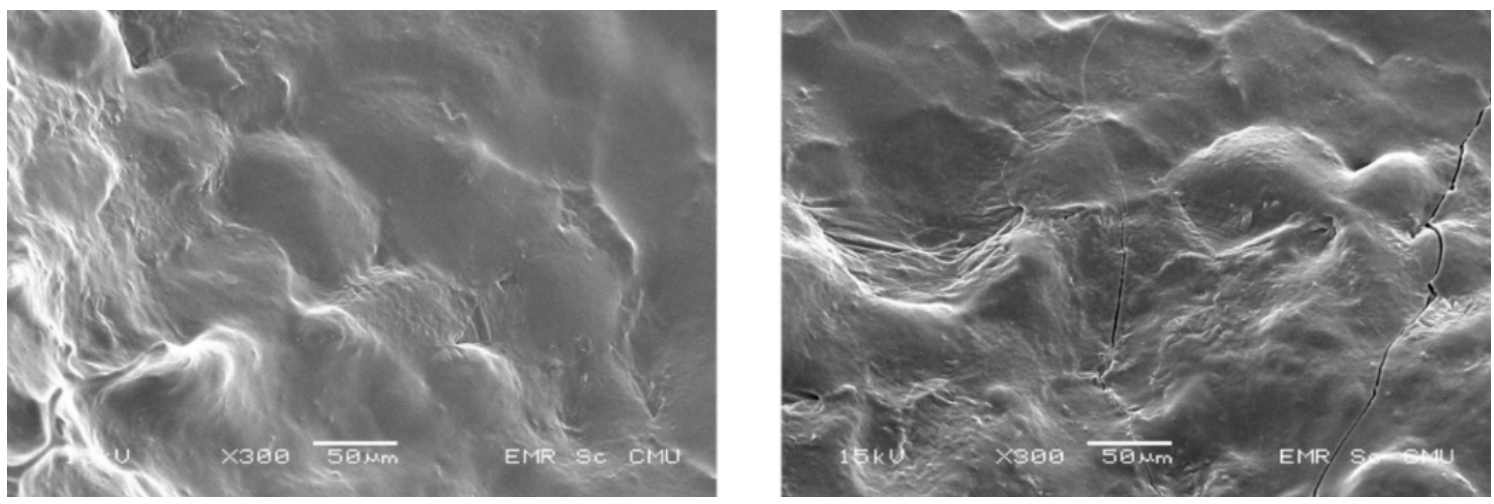

Figure 1 Internal structure of the texture of steamed glutinous rice kernels with a 300-magnification LVScanning Electron Microscope. 


\section{Streamed-cooked preparation}

Sample preparation for testing

Each soaking experiment used a 200 -g rice sample under different pressure and time conditions. These rice samples were subjected to 2 levels of pressure: Ambient pressure and gauge pressure of 1 bar using a standard pressure cooker that had been modified to allow pressure to be applied through its safety valve. There were various soaking times to be saturated. Before soaking, rice grains of the same weight were dipped and weighed to determine the amount of water that would remain on the surface of the rice. This was done to reduce errors in water content calculations within kernels. According to the results, the normal soaking rice method took $120 \mathrm{~min}$ and the pressure soaking method took $30 \mathrm{~min}$ for saturation to occur, as shown in Figure 2. After that, the 2 samples were steamed in a Thai Glutinous Rice Steamer. After cooking, the rice in the steamer was divided into 3 layers: Top, middle and bottom. This was done to account for any differences in the heat and pressure applied to them during the steaming process. The moisture content of each rice sample was determined using the gravimetric method. The sample was heated in a hot air oven at $105^{\circ} \mathrm{C}$ until it reached a constant weight. For each 5 replicates were carried out.

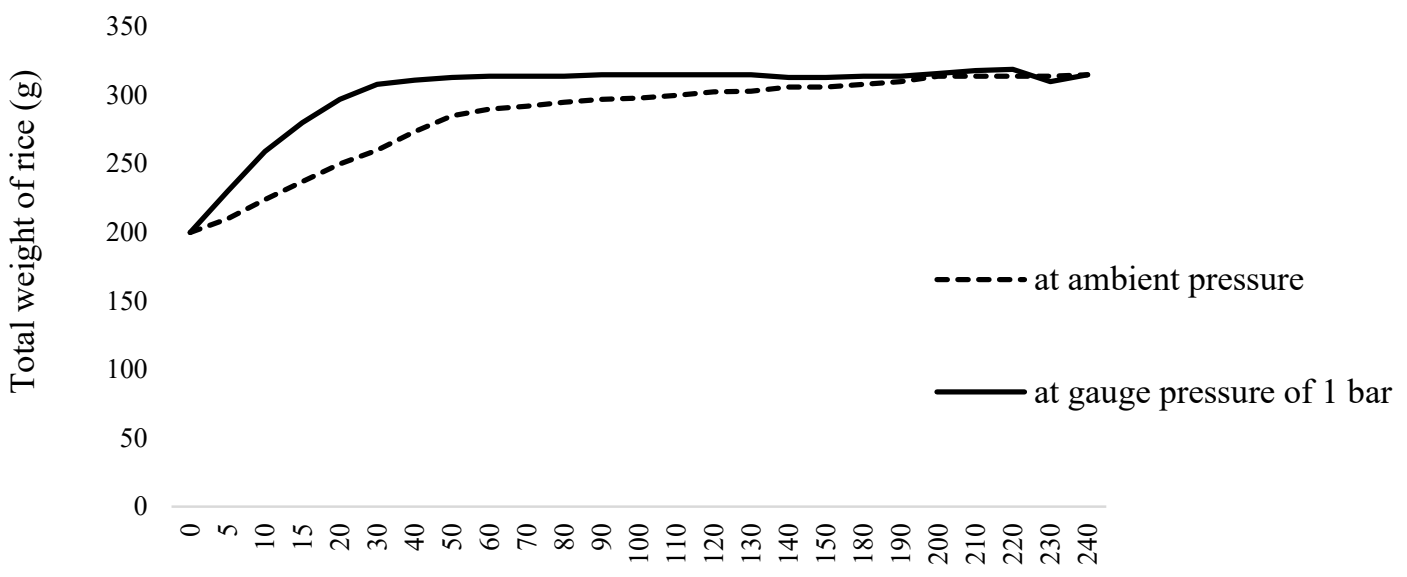

Soaking duration (minutes)

Figure 2 The relation of total rice weight and soaking duration.

\section{Experimental setup of compression test}

The main equipment used to measure mechanical characteristics of rice grain was a texture analyzer TA-XT-Plus (Manufactured by Stable Micro Systems ${ }^{\circledR}$ ), equipped with computer data processing as shown in Figure 3. The compression unit assembly of the texture analyzer consisted of a compression indenter (35-mm dia. cylindrical probe), a base (8-mm-thick transparent acrylic plate), a crosshead and a compression load cell. The compression indenter was connected to the crosshead with a 30-kg compression load cell. Test were carried out by compressing single grain of steamed-cooked glutinous rice on a flat rigid steel surface at $0.03 \mathrm{~mm} / \mathrm{s}$ [7]. During the operation, visual monitoring of the test was observed through the force-deformation curve simultaneously plotted on the screen of the computer. The compression test was stopped immediately after the travel distance of the indenter reached $98 \%$ of the grain thickness. The experiment was repeated for 15 grains and the average deformation curve was used in the analysis. A video recording controlled by Raspberry Pi Camera Module was used to monitor the contact area during compression. The camera was positioned directed under the 8-mm-thick transparent acrylic plate, where the rice was compressed against. The system was calibrated before each experiment. Recorded video files were converted into images in frame-by-frame basis. Image $\mathrm{J}$ was used to determine the contact area and dimension used to determine Poisson's ratio. Area corresponding to the initial yield point was used in Hooke's law equation presented previously. Compression force and contact area were used to compute normal stress on the grain. Deformation was used to compute the normal strain. The latter 2 were then used to compute moduli of elasticity. 


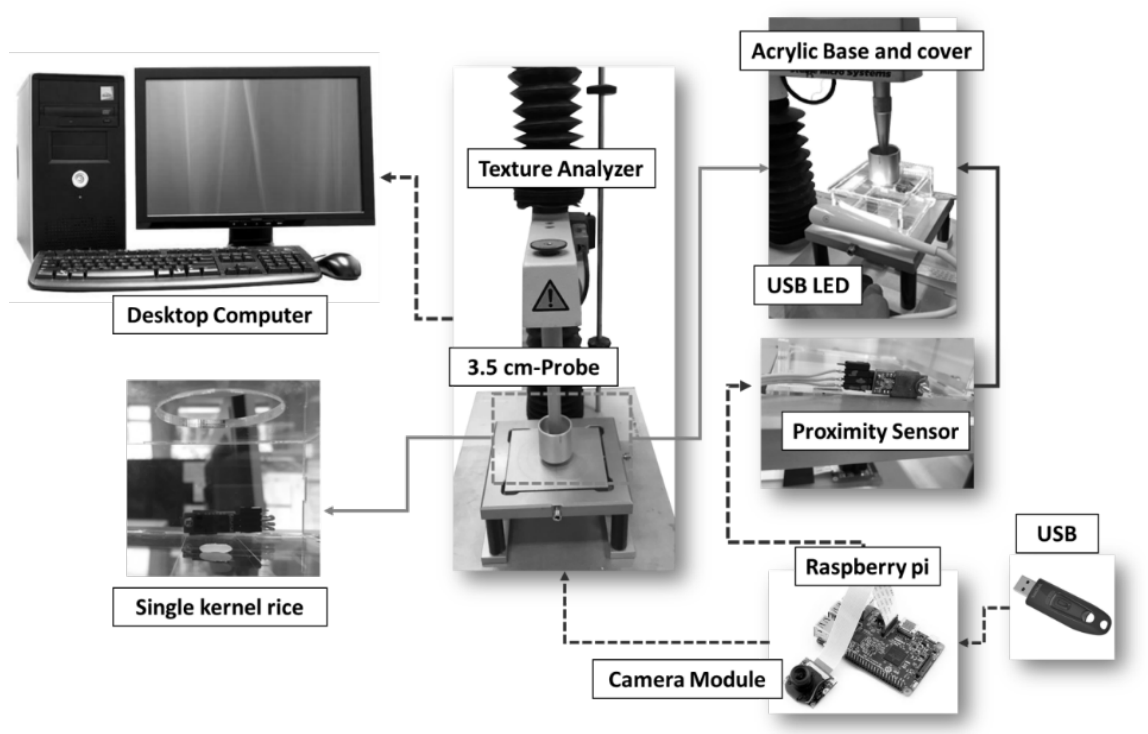

Figure 3 Texture analyzer and the compression unit assembly of the universal testing machine.

\section{Modulus of elasticity \\ Hooke's theory}

For ideal elastic materials, stress is directly proportional to strain and Young's modulus (E) based on Hooke's law is given by Eq. (1). Young's modulus is properly determined from the initial section of the stress-strain curve at relatively low deformation (before reaching the proportional limit). The constant of stress-strain relationship is called Modulus of Elasticity;

$\mathrm{E}=\frac{\sigma}{\varepsilon}=\frac{\Delta \mathrm{Pd}}{\Delta \mathrm{Az}}$

where, $E$ is Young's Modulus (MPa); $\sigma$ is stress $(\mathrm{MPa}) ; \varepsilon$ is strain (dimensionless), $\Delta P$ is change in compression force $(\mathrm{N}) ; \mathrm{d}$ is grain thickness $(\mathrm{mm}) ; \Delta A$ is change in contact area $\left(\mathrm{mm}^{2}\right)$; $\mathrm{z}$ is grain deformation $(\mathrm{mm})[5]$.

\section{Hertz's theory}

The second method of the apparent modulus of elasticity determination was achieved based on the Hertz equations for contact stresses applied in solid mechanics [7]. To calculate the apparent modulus of elasticity according to Hertz's theory, the Eq. (2) was used with assumption of small deformation and homogeneous elastic material;

$E=\frac{0.338 P\left(1-v^{2}\right)}{\mathrm{z}^{3 / 2}}\left[\mathrm{~K}_{\mathrm{U}}\left(\frac{1}{\mathrm{R}_{\mathrm{U}}}+\frac{1}{\mathrm{R}_{\mathrm{U}}^{\prime}}\right)^{\frac{1}{3}}+\mathrm{K}_{\mathrm{L}}\left(\frac{1}{\mathrm{R}_{\mathrm{L}}}+\frac{1}{\mathrm{R}_{\mathrm{L}}^{\prime}}\right)^{\frac{1}{3}}\right]^{3 / 2}$

where, $\mathrm{E}$ is Apparent modulus of elasticity; $\mathrm{P}$ is force $(\mathrm{N})$; $\mathrm{z}$ is grain deformation $(\mathrm{mm})$; $v$ is Poisson's Ratio; $R_{U}$ and $R_{U}^{\prime}$ are the maximum radius of curvature of the $R_{U}$ and $R_{L}$ as Figure 4 ; $K$ is Constant determined from elliptic integral tables; $d$ is grain thickness $(\mathrm{mm})$; $\mathrm{L}$ is grain length $(\mathrm{mm})$. 


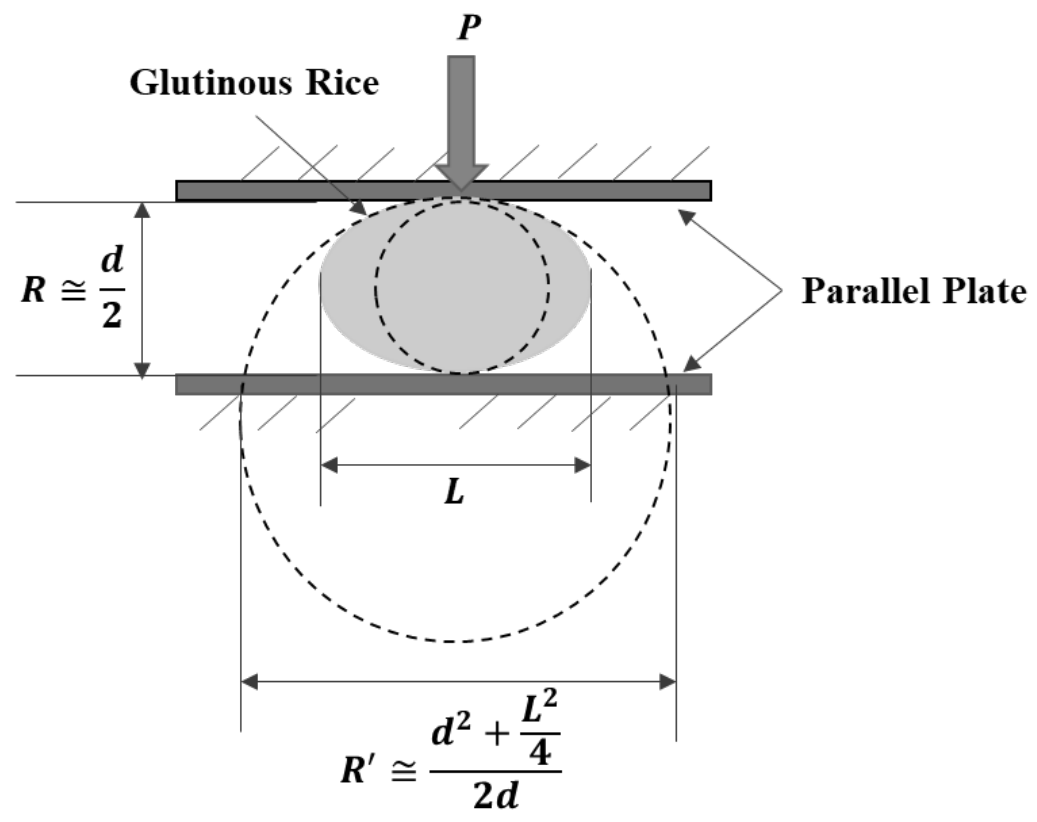

Figure 4 Estimation of minimum and maximum values for radius of curvature.

\section{Poisson's ratio}

Assuming steam-cooked glutinous rice has a smooth curved surface, its deformation (D) between 2 parallel flat plates and direct compression force $(\mathrm{P})$ can be expressed as follows by Sitkei [12] from the Eq. (3). Before testing, both the original length and diameter of specimens were recorded by using an image during compression. Axial displacement (strain) was measured and recorded by using the Texture analyzer TA-XT- Plus;

$v=\frac{\Delta \mathrm{D} / \mathrm{D}_{0}}{\Delta \mathrm{L} / \mathrm{L}_{0}}$

where, $v$ is Poisson's ratio; $\Delta D$ is transverse deformation (mm); $D_{0}$ is sample width (mm); $\Delta L$ is axial deformation $(\mathrm{mm}) ; L_{0}$ is sample length $(\mathrm{mm})$.

\section{Data management and calculation methods}

The modulus of elasticity is calculated by using Hooke's and Hertz's Theory. The parameters used in the Hooke's and Hertz's equations (Eqs. (1) and (2)) are evaluated by using ASAE Standard procedure. Following that, E values from 2 equations are compared to investigate the effect of Hooke's and Hertz's theories for estimating modulus of elasticity and appropriate theories for glutinous rice.

\section{Statistical analysis}

The modulus of elasticity and Poisson's ratio were calculated using the mean and standard deviation of the 5 replicates. Duncan's multiple range test was used to determine the significance of differences between treatment means at a $95 \%$ confidence level. 


\section{Results and discussion}

The physical and textural characteristics of the steamed-cooked glutinous rice samples at room temperature. The physical properties determined were grain length, width, thickness and contact area. Some of their physical properties of 2 varieties of rice were shown in Table 1. Found that steamed glutinous rice that has passed the ambient pressure immersion and gauge pressure of 1 bar in any position in steam-cooker has the same physical characteristics of the grain and not differ significantly. The shape of the grain does not depend on the position of the steam-cooker. Therefore, the rice can be used for sample in every position in steam-cooker. All parameters are used to calculate the modulus of elasticity based on Hertz's theory and Poisson's ratio.

The bottom grain has more gelatinization of rice than the middle and upper areas, the texture of the steam-cooked rice grain at the bottom receives more steam than the middle and upper areas. When heated, the hydrogen bonds within the starch molecules in the starch granules loosen and combine with the surrounding water, causing the polymer line of amylose and amylopectin packed in the starch granules to loosen and combine. As a result, starch granules have inflated and the viscosity of flour water has steadily increased, causing the grain to be heated more than other areas.

Contact area found that when the plate press in the grain rice, the contact area will be larger according to the deformation period by changing the contact area size of the grain to recorded video by Raspberry Pi Camera Module. Then the files were converted into images in frame basis. The result of image J was used to determine the contact area obtained follow Schneider's method [13] in Figure 5. From the relationship between the contact area and the deformation phase, which has an exponential relationship which is derived from finding the average contact area size of the cooked parboiled rice. Both methods are immersed.

Table 1 physical properties of 2 different soaking methods of rice.

\begin{tabular}{ccccc}
\hline Soaking method & $\begin{array}{c}\text { Length } \\
\mathbf{( m m})\end{array}$ & $\begin{array}{c}\text { Width } \\
\mathbf{( m m )}\end{array}$ & $\begin{array}{c}\text { Thickness } \\
\mathbf{( m m )}\end{array}$ & $\begin{array}{c}\text { Contact Area } \\
\left(\mathbf{m m}^{\mathbf{2} \times \mathbf{1 0}^{-6} \mathbf{)}}\right.\end{array}$ \\
\hline Ambient pressure & $8.16 \pm 0.23^{\mathrm{a}}$ & $2.36 \pm 0.07^{\mathrm{a}}$ & $1.98 \pm 0.08^{\mathrm{a}}$ & $1.75 \pm 0.04^{\mathrm{a}}$ \\
\hline Gauge pressure of 1 bar & $7.99 \pm 0.24^{\mathrm{a}}$ & $2.51 \pm 0.08^{\mathrm{a}}$ & $2.00 \pm 0.04^{\mathrm{a}}$ & $1.94 \pm 0.10^{\mathrm{b}}$ \\
\hline
\end{tabular}

Mean \pm SD is given in the table. The difference letters $(a, b)$ in the same column mean that the mean values are differ significantly at a confidence level of $95 \%(p<0.05)$.
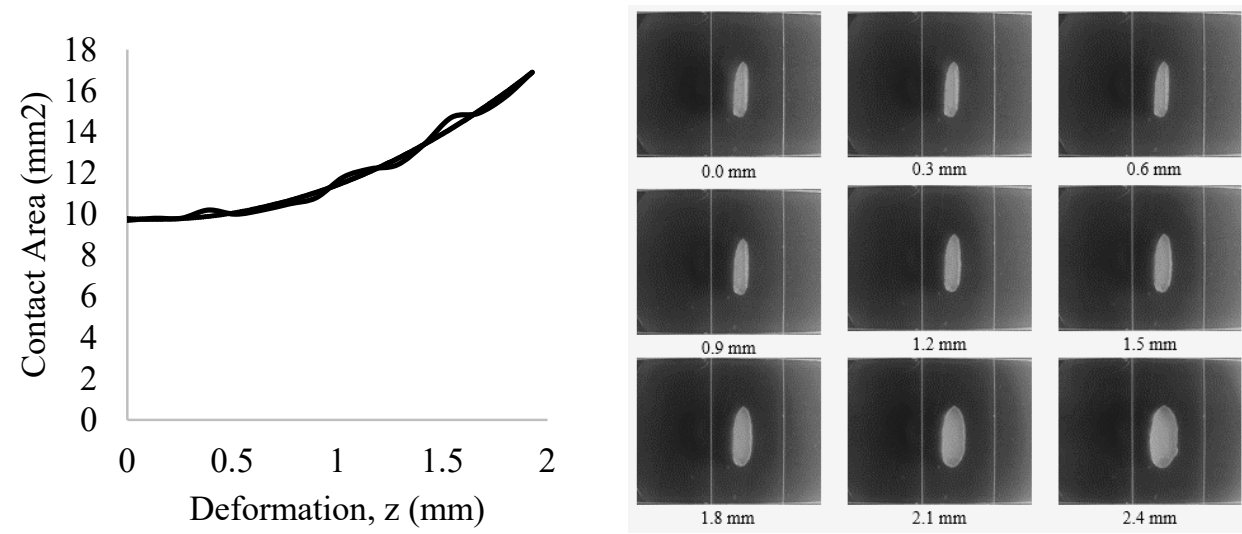

Figure 5 Variation of contact area with deformation for glutinous rice.

\section{Relationship between compression force and deformation}

The Figure 6 shows the force-deformation diagram of the normal soaking rice and pressure soaking rice compressed in their steamed-cooked state. Found that the deformation behavior of steamed glutinous rice kernels is nonlinear. Therefore, it can divide the relationship between stress and stress for 3 stages from the difference of slope. 


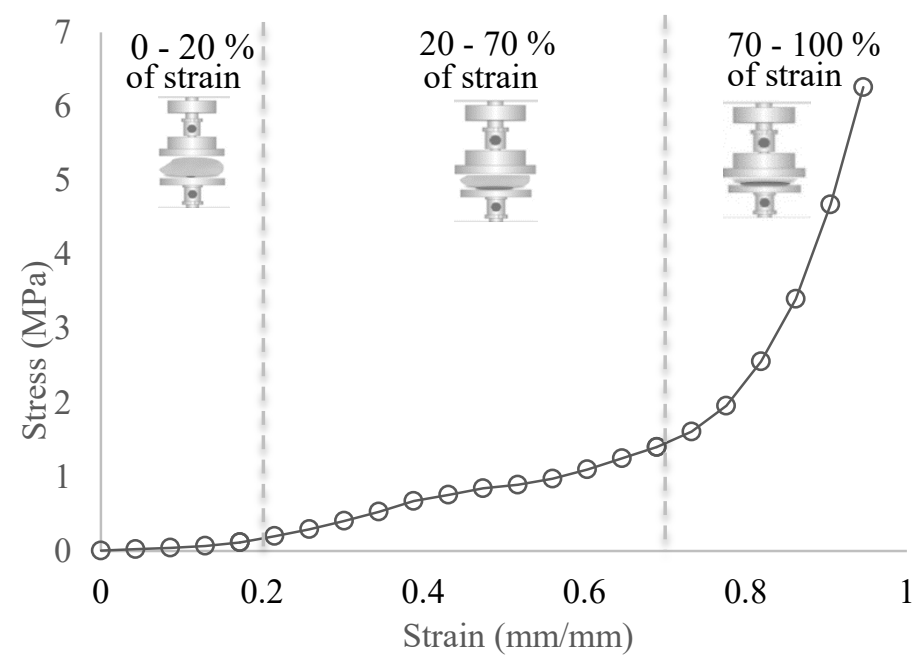

Figure 6 The relationship between stress and strain for 3 range of glutinous rice.

The surface of the rice grain is compressed by a force perpendicular to some of the rice because the rice grains have a rough surface, resulting in uneven force in the first stage, which is in the range of $0-20 \%$ of stress. The second stage has a stress level of $20-70 \%$. The second stage of the slope has more sleepers than the first stage. The rice kernel was compressed through the workpiece. The stress and strain are proportional to each other, and the slope is constant, implying that the behavior is elastic. The researcher chose this period to study the mechanical properties of steamed glutinous rice kernels, according to Hooke's theory. The third stage is the period at 70 percentage onwards. The force exerted on the specimen has increases and make the steamed glutinous rice kernels are tightly compressed until broken.

Comparison of modulus of elasticity of Glutinous rice from 2 soaking methods determined based on Hooke's theory and Hertz's theory

The modulus of elasticity and Poisson's ratio were determined as mechanical properties. Based on the regression method, modulus of elasticity values was calculated as the slope of the linear part of the stress-strain curve as follow by Dowling [14]. The equations were used to calculate stresses and strains (1) The equations were used to calculate Young's Modulus according to Hooke's theory and modulus of elasticity according to Hertz's theory (2). Figure 7 represents the analysis of the elastic modulus from Hooke's and Hertz's theories

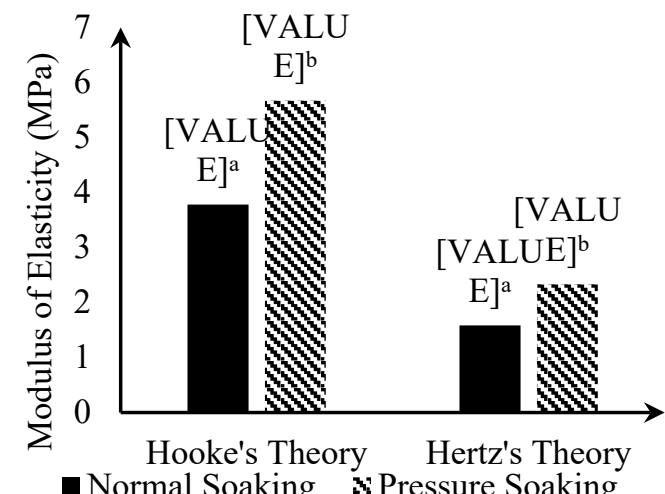

- Normal Soaking Pressure Soaking

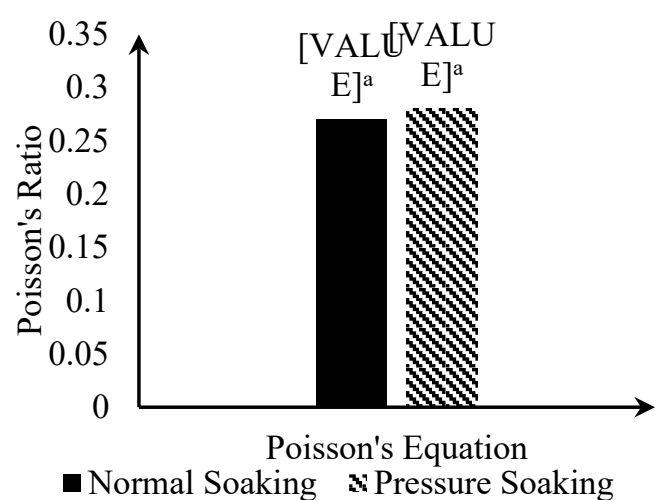

- Normal Soaking \$ Pressure Soaking

Figure 7 Mechanical properties of 2 different soaking methods of rice. 
Based on Hooke's theory, the modulus of elasticity of steamed glutinous rice kernels that were treated by under pressure and ambient pressure soaking was 5.67 and $3.78 \mathrm{MPa}(p>0.05)$, respectively. For Hertz's theory, the elastic modulus varies approximately 1.5 times and differ significant $(p<0.05)$. The modulus of elasticity of steamed glutinous rice kernels that were treated by under pressure and ambient pressure soaking was 2.33 and $1.58 \mathrm{MPa}(p>0.05)$, respectively. The elastic modulus varies approximately 1.5 times and differ significant $(p<0.05)$. In the case of soaking under pressure is higher than ambient pressure because of difference water absorption of the rice from each method of soaking. The rice grain that are saturated with water by ambient pressure soaking methods was complete gelatinization than gauge pressure of 1 bar soaking as shown in Figure 8, therefore, the rice grain was softer.

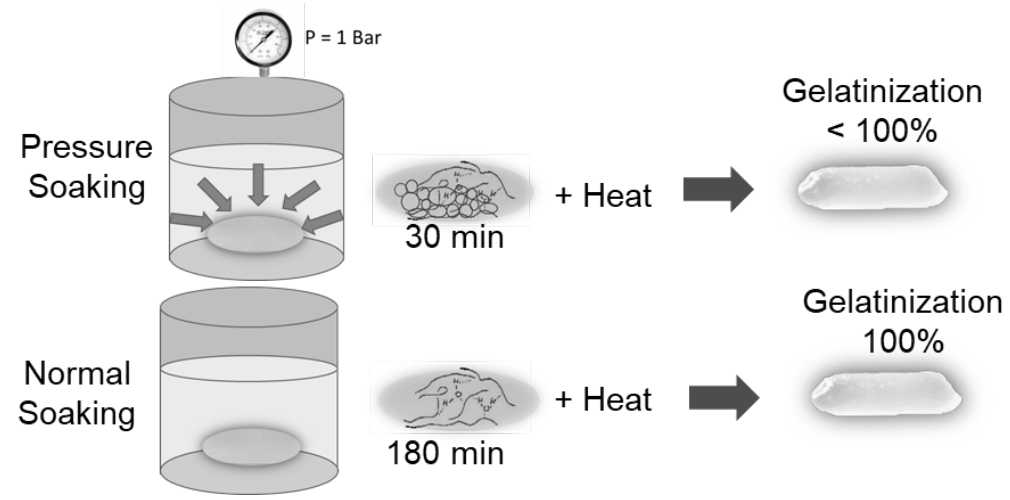

Figure 8 Gelatinization of Pressure gauge of 1 bar and ambient pressure soaking glutinous rice

\section{theory}

Comparison of modulus of elasticity of Glutinous rice using Hooke's theory and Hertz's

Modulus of elasticity of glutinous rice by several soaking methods. The method of test is indicated as places the grain in flat position between parallel plates and get the parameter such as force, deformation, shape and Poisson's ratio to calculate the modulus of elasticity follow Hooke's and Hertz's theory found that modulus of elasticity of steamed glutinous from Hooke's theory was about 2.4 times higher than Hertz's theory $(p<0.05)$ which has a significant difference.

Hooke theory uses variables to calculate the elastic modulus, including force, deformation, and contact area centred on Hooke and Hertz's equations. Hertz's theory makes use of variables such as force, deformation, Poisson's ratio, and grain curvature radius. The contact area, Poisson's proportions, and curvature radius have all increased. The modulus of elasticity decreased. Given the shape of the rice grain, the surface is smooth and appears cylindrical. So, the curvature radius was smaller change while compressed and resulting in a high modulus of elasticity in contrast to contact area that changes more while being pressed. Therefore, as a result of modulus of elasticity based on Hooke's theory is higher than Hertz's theory.

\section{Poisson's ratio of Glutinous rice}

As previously discussed, using Hertz's theory to determine elastic modulus is required to know Poisson's ratio. As a result, many researchers have investigated Poisson's ratio for agricultural produce. Poisson's ratio equation was used in a study on glutinous rice. Many researchers have also assumed that the variation of Poisson's ratio was 0.2 to 0.5 for Poisson's ratio of agricultural produce. Kohayashe [15], with Kawamura [16] assuming a Poisson's ratio of 0.21 and Yamagushi [17] assuming a Poisson's ratio of 0.25. As a result, Poisson's ratio was 0.27 , which was similar to the result of another investigation.

\section{Conclusions}

Modulus of elasticity of steamed-cooked Thai Sanpatong 1 rice was studied based on 2 theories of elasticity, Hooke's and Hertz's theory. Calculating modulus of elasticity of rice kernels obtained from Hertz's theory yields a higher modulus of elasticity. In the case of rice grains, modulus of elasticity calculation using Hooke's theory is more practical than that using Hertz's theory. This usability of Hooke's theory is noticeable because complex parameters such as such Poisson's ratio and grain radius of 
curvature are not required. Both of the modulus of elasticity calculated using these 2 theories reveal that the rice grains soaked under gauge pressure of 1 bar is stiffer than that ambient soaked. Although soaking rice under pressure takes less time than normal soaking, the cookers have to recognize that the rice grains will be stiffer, which may not be desired by consumers. The mechanical properties obtained from this study can also be used to simulate rice deformation under chewing. These simulations will allow us to study the forces system in the mouth during chewing. This force system can be used for comparing and confirming the results of sensory test.

\section{Acknowledgements}

This research was supported by grants funded by Faculty of Engineering, Chiang Mai University, Thailand and the authors thank the Faculty of Agro-Industry, Chiang Mai University, Thailand for providing instruments and laboratory facilities.

\section{References}

[1] A Ahromrit, DA Ledward and K Niranjan. High pressure induced water uptake characteristics of Thai glutinous rice. J. Food Eng. 2006; 72, 225-33

[2] Iwasaki. Measures for the enhancement of rice consumption and diversification of rice utilization. In: Proceedings of the International Seminar on the Diversification of Rice Utilization, Bangkok, Thailand. 1987.

[3] D Mindo. Sensory assessment of cooked milled rice. In: Proceedings of the workshop on chemical aspects of rice grain guality, Los Banos, Laguna, Philippines. 1979, p. 313-26.

[4] CM Perez, BO Juliano and MC Bourne. Measuring hardness distribution of cooked rice by single grain puncture. J. Texture Stud. 1996; 27, 1-13.

[5] D Shitanda, Y Nishiyama and S Koide. Compressive strength properties of rough rice considering variation of contact area. J. Food Eng. 2001; 53, 53-8.

[6] W Burubai, E Amula, RM Davies, GWW Etekpe and SP Daworiye. Determination of Poisson's ratio and elastic modulus of African nutmeg (Monodora myristica). Int. Agrophys. 2008; 22, 99102.

[7] American Society of Agricultural Engineers. Compression test of food materials of convex shape. American Society of Agricultural Engineers, Michigan, 2004, p. 585-92.

[8] S Bamrungwong, T Satake, D Vargas and S Yoshizaki. Fundamental studies on mechanical properties of long grain rice varieties. J. Jpn. Soc. Agr. Mach. 1987; 31, 232-40.

[9] KDK Mostafa, M Hossein and M Saeid. Determination of Poisson's ratio and young's modulus of red bean grains. J. Food Proc. Eng. 2009; 34, 1573-83.

[10] R Osvaldo. Mechanical properties of rough and dehulled rice during drying. Int. J. Food Stud. 2013; 2, 158-66.

[11] S Manizhe, G Davoud and G Mahdi. Measurement and evaluation of the apparent modulus of elasticity of apple based on Hooke's, Hertz's and Boussinesq's theories. J. Food Eng. Meas. 2014; 54, 133-9.

[12] G Sitkei. Mechanics of agricultural materials. Elsevier, Hungary, 1986, p. 114-85.

[13] CA Schneider, WS Rasband aand KW Eliceiri. NIH Image to ImageJ: 25 years of image analysis. Nat. Meth. 2012; 9, 671-5.

[14] NE Dowling. Engineering methods for deformation, fracture, and fatigue. Pearson Education Limited, London, 2012, p. 190-213.

[15] H Kohayashe, Y Miwa and T Torii. Studies on compression and shearing tests of brown rice. In: Proceedings of the $35^{\text {th }}$ Annual Meeting of the Japanese Society of Agricultural Machinery and Food Engineers. 1976, p. 101.

[16] N Kawamura, H Horio and Y Sasaki. Mechanical properties of rice ear and grain. Jpn. Soc. Agr. Mach. 1968; 30, 88-92.

[17] S Yamaguchi, S Yamazawa and K Wakabayashi. On relaxation modulus of rice endosperm. J. Jpn. Soc. Agr. Mach. 1981; 43, 239-45. 